\title{
Cochrane
}

Cochrane Database of Systematic Reviews

\section{Interventions for improving medical students' interpersonal communication in medical consultations (Protocol)}

Gilligan C, James EL, Snow P, Outram S, Ward BM, Powell M, Lonsdale C, Cushing AM, Silverman J, Regan T, Harvey P, Lynagh MC

Gilligan C, James EL, Snow P, Outram S, Ward BM, Powell M, Lonsdale C, Cushing AM, Silverman J, Regan T, Harvey P, Lynagh MC. Interventions for improving medical students' interpersonal communication in medical consultations.

Cochrane Database of Systematic Reviews 2016, Issue 11. Art. No.: CD012418.

DOI: 10.1002/14651858.CD012418.

www.cochranelibrary.com 
TABLE OF CONTENTS

HEADER . . . . . . . . . . . . . . . . . . . . . . . . . . . . . . . . . . . 1

ABSTRACT . . . . . . . . . . . . . . . . . . . . . . . . . . . . . . . . . . . . . . . 1

BACKGROUND . . . . . . . . . . . . . . . . . . . . . . . . . . . . . . . . . . . .

OBJECTIVES . . . . . . . . . . . . . . . . . . . . . . . . . . . . . . . . . . . . . . . . . .

METHODS . . . . . . . . . . . . . . . . . . . . . . . . . . . . . . . . . . . . . . . .

ACKNOWLEDGEMENTS . . . . . . . . . . . . . . . . . . . . . . . . . . . . . . . . . . . . . . . . . . . .

REFERENCES . . . . . . . . . . . . . . . . . . . . . . . . . . . . . . . . . . . . . 9

APPENDICES . . . . . . . . . . . . . . . . . . . . . . . . . . . . . . . . . . . . . 12

CONTRIBUTIONS OF AUTHORS . . . . . . . . . . . . . . . . . . . . . . . . . . . . . . . . . . . . . . .

DECLARATIONS OF INTEREST . . . . . . . . . . . . . . . . . . . . . . . . . . . . . . . 13

NOTES . . . . . . . . . . . . . . . . . . . . . . . . . . . . . . . . . . . . . . . . 14

Interventions for improving medical students' interpersonal communication in medical consultations (Protocol)

Copyright $\odot 2016$ The Cochrane Collaboration. Published by John Wiley \& Sons, Ltd. 


\section{[Intervention Protocol]}

\section{Interventions for improving medical students' interpersonal communication in medical consultations}

Conor Gilligan $^{1}$, Erica L James ${ }^{1}$, Pamela Snow ${ }^{2}$, Sue Outram ${ }^{1}$, Bernadette M Ward ${ }^{3}$, Martine Powell ${ }^{4}$, Chris Lonsdale ${ }^{5}$, Anne M Cushing $^{6}$, Jonathan Silverman ${ }^{7}$, Tim Regan ${ }^{8}$, Pam Harvey ${ }^{3}$, Marita C Lynagh ${ }^{1}$

${ }^{1}$ School of Medicine and Public Health, University of Newcastle, Hunter Medical Research Institute, Callaghan, Australia. ${ }^{2}$ School of Psychology and Psychiatry, Monash University, Bendigo, Australia. ${ }^{3}$ Rural Health, Monash University, Bendigo, Australia. ${ }^{4}$ Centre for Investigative Interviewing, School of Psychology, Deakin University, Geelong, Australia. ${ }^{5}$ Institute for Positive Psychology and Education, Australian Catholic University, Strathfield, Australia. ${ }^{6}$ Institute of Health Sciences Education, Barts and The London School of Medicine and Dentistry, London, UK. ${ }^{7}$ School of Clinical Medicine, University of Cambridge, Cambridge, UK. ${ }^{8} \mathrm{Hunter}$ New England Population Health, Hunter New England Local Health District, Wallsend, Australia

Contact address: Conor Gilligan, School of Medicine and Public Health, University of Newcastle, Hunter Medical Research Institute, Callaghan, NSW, 2308, Australia. Conor.Gilligan@newcastle.edu.au.

Editorial group: Cochrane Consumers and Communication Group.

Publication status and date: New, published in Issue 11, 2016.

Citation: Gilligan C, James EL, Snow P, Outram S, Ward BM, Powell M, Lonsdale C, Cushing AM, Silverman J, Regan T, Harvey P, Lynagh MC. Interventions for improving medical students' interpersonal communication in medical consultations. Cochrane Database of Systematic Reviews 2016, Issue 11. Art. No.: CD012418. DOI: 10.1002/14651858.CD012418.

Copyright (C) 2016 The Cochrane Collaboration. Published by John Wiley \& Sons, Ltd.

\section{A B S T R A C T}

This is a protocol for a Cochrane Review (Intervention). The objectives are as follows:

To assess the effects of interventions for medical students that aim to improve interpersonal communication in medical consultations.

\section{B A C K G R O U N D}

\section{Description of the condition}

The importance of effective communication between clinicians and patients was highlighted in the Institute of Medicine's Crossing the quality chasm report (Richardson 2001). This report proposed free and open sharing of knowledge between patients and clinicians as 1 of 10 principles for redesigning the healthcare system to ensure delivery of optimal patient-centred care. Effective doctor-patient communication has been shown to affect patient satisfaction (Pollak 2011; Street 2009), decision making (NHS 2010), treatment adherence (Street 2009; Zolnierek 2009), a range of patient outcomes such as blood pressure and emotional health (Stewart 1995b), and doctors' job satisfaction (Maguire 2002). The information gathered as part of the medical history is critical to the establishment of an accurate diagnosis, with most information used to form a diagnosis gathered in this step (Peterson 1992). Subsequent steps of the medical consultation facilitate patient education and shared decision-making, with associations established between patient-centred communication, therapeutic alliance, and adherence to treatment (Pinto 2012; Thompson 2016). It has been estimated that a medical doctor will conduct approximately 200,000 medical consultations during his or her working career (Silverman 2013). Thus, it is incumbent upon educators that medical students are appropriately trained to ensure that, upon graduating, these 
consultations are conducted effectively using appropriate patientcentred communication (Simpson 1991).

Medical consultations should be a joint, collaborative effort between doctor and patient, using patient-centred communication to acknowledge and understand the patient's desires for information, shared decision-making, and discussions of care (Stewart 2001). While global agreement on definitions of the terms is elusive, there is broad agreement about the concepts and importance of patient-centred care and patient-centred consultation. These concepts are integral to the display of respect for patients, and thus, along with patient involvement in care systems, should be regarded as an ethical and democratic right (Gregory 2007). Definitions generally include elements of a biopsychosocial perspective of illness; consideration of each individual's personal meanings of illness; being sensitive to patients' preferences for information and shared decision-making; and developing a therapeutic relationship between doctor and patient (Mead 2002).

Patient-centred medical consultations call upon a set of skills that are considered both teachable and learnable by medical professionals at any point on their career trajectory (Aspegren 1999). We will use the term 'interpersonal communication' to refer to these skills which facilitate patient-centred communication and care. Interpersonal communication can be defined as communication that occurs from one individual to another (dyadic or small group), is non-mediated (face-to-face), and is shaped by the individual characteristics, social roles and relationships of the people involved (Hartley 1999). Interpersonal communication is the process by which we establish a communicative relationship and exchange messages to establish shared goals and understandings (Burleson 2010; Hargie 2011). We will refer to communication interventions as those aiming to improve the skills associated with such communication.

Significant progress has been made in the development and evaluation of formal curricula for interpersonal communication in medicine (Aspegren 1999; Smith 2007). Skills in interpersonal communication in clinical contexts are recognised as being different from everyday communication skills and should be developed through careful teaching and experiential learning (Benbassat 2009; Silverman 2013). Descriptive data suggest that students find acquiring skills in interpersonal communication challenging (Lumma-Sellenthin 2009; Royston 1997) for reasons including difficulty relearning or reconditioning engrained communication styles (Macdonald 2002), difficulty attending to medical and psychosocial needs simultaneously (Aper 2015), lack of exposure to models of patient-centred care (Thistlethwaite 1999), and wide variability between clinical and non-clinical role models (Rees 2002). With an absence of up-to-date, high-quality systematic reviews, evidence on the effects of communication curricula for improving medical students' skills in conducting effective patientcentred consultations and in improving clinical practice is unclear. The increasing demand for medical students to be specially trained to communicate effectively and efficiently has seen the emer- gence of skills associated with interpersonal communication as core graduate competencies in medical training around the world (Australian Medical Council 2012; General Medical Council 2015; Health Professions Council of South Africa 2014; Laidlaw 2009). New doctors are required to possess a range of skills for communicating in different formats such as face-to-face, online and by telephone, and different medical contexts, such as doctorpatient consultations, communicating about the patient (e.g. with other medical professionals), and communicating about medicine and science in general (e.g. lectures and conferences). In this review, we will focus on the medical consultation, referring to the verbal and non-verbal interaction between physician and patient that occurs during face-to-face encounters. The medical consultation has been described using a range of models, all of which summarise the process as including elements of relationship building, information gathering, information giving, and treatment planning which can occur in both initial and follow-up encounters (Keller 1994; Kurtz 1998; Makoul 1998; Novack 1992; Stewart 1995a). The overall goal of such encounters is a shared understanding of issues and plans, while the specific goals of any individual consultation can vary from diagnosis to an understanding of cause, risk, prognosis, the benefits and risks of various treatment options, health behaviour change, screening and any number of other therapeutic and health promoting activities.

\section{Description of the intervention}

\section{Models of the medical consultation and communication training}

Teaching and assessment of interpersonal communication have been guided by frameworks and models evolving over several decades (reviewed in Boon 1998). To the core elements of relationship establishment, information gathering, and patient education (Lipkin 1995) have been added the need for the doctor to gather information about the patient's understanding of his or her own health (Makoul 2001a), recognition of the influence of clinicians' personal experiences on their interpersonal communication (Windover 2014), and the need to incorporate the electronic health record (Duke 2013). While the most recent studies have incorporated the use of technology, the fundamental structure and content of the models has not changed since the establishment of the Kalamazoo Consensus Statement in 2001 (Makoul 2001b). The Kalamazoo Consensus drew upon five contemporary models for doctor-patient communication; namely the Bayer Institute for Health Care Communication E4 Model (Keller 1994), Three Function Model/Brown Interview Checklist (Cole 2013; Novack 1992), the Calgary-Cambridge Observation Guide (Kurtz 1998), patient-centered clinical method (Stewart 1995b), and the SEGUE Framework for teaching and assessing communication skills (Makoul 1998). It brought together a comprehensive set 
of skill competencies (Makoul 2001b). Specific communication tasks as well as knowledge, skills, and attitudes associated with the following essential elements are listed in the consensus statement: build the relationship, open the discussion, gather information, understand the patient's perspective, share information, reach agreement on problems and plans, and provide closure.

Subsequent to the synthesis of communication tasks in the Kalamazoo Consensus Statement, Kurtz and colleagues expanded their Calgary-Cambridge guide to more clearly connect with the process of doctor-patient consultations (Kurtz 2003). This clarified and expanded upon the specific skills used at each step of the medical consultation process. For example, when gathering information, necessary skills include using open and closed questions appropriately, structuring, clarifying and summarising information, picking up verbal and nonverbal cues from the patient, facilitating patients' responses verbally and non-verbally, and listening attentively. The marriage of process and content proposed by Kurtz and colleagues represents a true amalgamation of the communication skills (performance of specific tasks and behaviours) with the interpersonal skills (relational, process-oriented skills such as respect, empathy, and considering the patient's perspective) required to establish a therapeutic relationship (Kurtz 2003; Makoul 2001b). In this review we will include all interventions for medical students that specifically target the skills associated with what we have defined as interpersonal communication. These skills are likely to include: the appropriate use of open and closed questions, active listening, picking up on verbal and nonverbal cues, facilitating patients' responses, eliciting patients' concerns, considering the patient's ideas, concerns and expectations (gathering and understanding their perspective), working in partnership with the patient to explain and plan, and make shared decisions, maintaining structure of the consultation, clarifying and summarising information.

\section{Training methods}

Interperonal communication training for medical students takes a range of forms depending upon the resources available, the current training level of students (e.g. undergraduate degree, postgraduate training program), and the context of learning (on campus, clinical placement, online). Interventions can be categorised as being delivered face-to-face or in self-directed formats. Face-to-face interventions are typically delivered as lectures for large groups of students or workshops for small groups. The latter can provide opportunities for participants to practice communication with real or simulated patients or their peers. They also enable provision of feedback from peers, facilitators and/or patients (whether real or simulated). These interventions may be based around live roleplays or feedback on videotaped consultations (Deveugele 2005; Maguire 1986). Self-directed interventions are those where the learner receives individual training using written or audiovisual materials, either in hard copy or in online or e-learning format such as online video demonstrations (Cook 2010).

\section{Assessment of interpersonal communication}

The Kalamazoo Consensus outlines three methods of assessing interpersonal communication: checklists (observer ratings); patient surveys; and examinations (of knowledge and perceptions using traditional written questions or questions linked to stimulus such as a video vignette). Both checklists and patient surveys can be used in the assessment of interactions with real or simulated patients, can occur live or based on recorded interactions, and can be used in formative or summative assessments such as Objective Standardised Clinical Examinations (OSCE) (Duffy 2004). Given the variation in validity and reliability among techniques, the assessment method utilised affects the capacity to compare different intervention studies. In this review we will categorise each study based on the method of assessment (observer ratings or survey/ examination scores) and the nature of the observed consultation (real patient, simulated patient, live, recorded). Given our focus on behaviour change, we will not include data obtained through student examinations or surveys. Where studies use more than one of the included methods of assessment, data for each outcome will be extracted and compared to other outcome data as appropriate.

\section{How the intervention might work}

Interventions to improve the interpersonal communication of medical students aim to produce doctors capable of delivering effective, safe and patient-centred health care when they enter the workforce. Education-based interventions work by bringing about change in learners' attitudes, increasing their knowledge, and importantly, increasing their competence in performing particular skills. In the case of medical consultation skills, educational interventions are likely to improve learners' skills and knowledge through: modelling by and feedback from educators; and experiential learning, with opportunities to practice, reflect, and receive constructive feedback, draw upon knowledge and previous experience, and learn in a self-directed fashion (Kaufman 2003). While the highest level of evaluation of learning is the application of skills in clinical practice leading to improved patient outcomes, the only immediately measurable outcomes for undergraduate students are improvements in skill, knowledge, attitudes, and confidence (Kirkpatrick 1996; Naugle 2000; Smidt 2009). Student learning outcomes can be conceptualised hierarchically (Alliger 1989). At the very least, participation in a communication intervention should increase knowledge of patient-centred approaches to communication. Next, these interventions should increase confidence in undertaking effective doctor-patient consultations. However, the ultimate goal of communication interventions should always be to improve actual behavioural skills to undertake evidence-based doctor-patient consultations. These behavioural outcomes are assessable using the methods outlined in 
the Kalamazoo Consensus Statement and form the basis of the outcome measures assessed in this review.

\section{Why it is important to do this review}

Given community and professional concerns regarding the physical, emotional and financial impact of poor communication by medical practitioners, there is a critical need to evaluate communication training programs in medical education. To date, the effectiveness of interventions for improving medical students' interpersonal communication has not been demonstrated unequivocally. Moreover, there is significant variability in communication curricula across medical schools (Hargie 2010; Hoffman 2004) and new methodologies have become increasingly popular since the publication of previous reviews (Lanken 2015). Given the rapid evolution of innovative teaching and learning approaches, it is timely to review the effectiveness of approaches which have been utilised. Thus there is a need to determine:

1. the evidence base for communication interventions for medical students;

2. the teaching and learning approaches associated with improvements in medical students' interpersonal communication;

3. the most effective approaches to teaching medical communication in the context of pragmatic limitations of medical curricula; and

4. gaps in knowledge about communication interventions for medical students to guide future teaching and research endeavours.

This review will aim to provide necessary guidance to medical educators and medical education accrediting bodies regarding the most effective communication programs in medical curricula, and identify the necessary resources for teaching these programs.

\section{Determining the evidence base for communication interventions for medical students}

A number of completed and ongoing reviews have sought to examine the effectiveness of communication training programs in medicine (Aspegren 1999; MacDonald-Wicks 2012; Smith 2007; Van Nuland 2005). Aspegren 1999 reviewed 83 randomised, quasi-randomised, and non-randomised trials and descriptive studies of communication training for medical students and concluded that teaching interpersonal communication to medical students can improve the students' ability to undertake doctor-patient consultations. However, the methodological quality of included studies was not adequately assessed, and the inclusion of non-randomised trials and descriptive studies limited the extent to which improvements in interpersonal communication could be attributed to the interventions described. Smith 2007 identified 24 randomised controlled trials (RCTs) that were available from 1977 to 2005 and conducted meta-analyses on 15 that met their inclusion criteria. Smith 2007 only included RCT interventions; however, given the settings in which these interventions are expected to be delivered (e.g. university classrooms, hospital clinics), it is possible that other study designs (such as cluster and quasiRCTs) may also be relevant. In addition, a preliminary search of the research undertaken since 2005 suggests at least 30 additional RCTs of communication training for medical students have been published since the Smith 2007 review.

The authors of a planned Cochrane review (Van Nuland 2005) intend to assess the effects of communication training programs specifically for general practice (GP) trainees. GP trainees are completing their training for specialisation, and as such, have advanced in their training beyond the basic medical degree. Furthermore, Van Nuland 2005 will exclude studies that include undergraduate students. The authors of MacDonald-Wicks 2012 are reviewing the effectiveness of assessment tools and methods for teaching interpersonal communication to students in the health professions. Their review includes students from undergraduate and postgraduate medical, nursing, and allied health programs, including nutrition, dietetics, occupational therapy, physiotherapy, among others. Given the heterogeneous nature of professional roles and scope of practice, undergraduate training programs, and student cohorts, it is important to examine medical education separately.

The review by Smith 2007 demonstrated that providing structured feedback on participants' performance, and engaging in small group discussions were associated with larger improvements in skills compared to other methods (e.g. lectures, clerkship experience, assigned readings). An overview of systematic reviews of strategies for teaching communication skills to qualified doctors (Berkhof 2011) also reported little evidence for interventions based on lectures, or those based on modelling appropriate interpersonal communication to participants. Like the review by Smith and colleagues, stronger evidence was reported for interventions based on role-plays and feedback from educators, particularly when used in combination with self-directed didactic techniques (e.g. written information, reviewing videos).

Our review will differ from these in a number of ways. First, we will ensure methodological rigour by appropriately reviewing identified studies with careful consideration of research design and additional features of methodological quality. Second, we will focus on students completing an undergraduate or graduate-entry medical degree which will help to ensure that we know what works for these students, as compared with more refined samples completing a medical specialisation (e.g. Van Nuland 2005), or students in other allied health programs (e.g. MacDonald-Wicks 2012). Third, identifying the intervention characteristics associated with improvements in interpersonal communication will help to focus the development of future curricula.

\section{O B J E C T IVES}


To assess the effects of interventions for medical students that aim to improve interpersonal communication in medical consultations.

\section{METHODS}

\section{Criteria for considering studies for this review}

\section{Types of studies}

We will include:

- randomised controlled trials (RCTs);

- cluster RCTs;

- non-randomised controlled trials including quasi-RCTs (where randomisation is attempted but is inadequate, such as allocation by day of the week or date of birth).

\section{Types of participants}

We will only include interventions for medical students. We define medical students as people who are enrolled in an undergraduate or graduate-entry medical degree. Where studies include participants from multidisciplinary courses, we will only include studies where subgroup analyses allow data for medical students to be identified and extracted separately.

We will exclude programs delivered as continuing medical education or postgraduate programs delivered to registered professionals. Students from other allied healthcare disciplines, such as nursing, physiotherapy, pharmacy, and psychology, will be excluded. We will not exclude studies on the basis of the age of students, country, setting (classroom, hospital), frequency of intervention contact, duration/dose of intervention, timing, skills targeted, or medical system in which the intervention is delivered.

\section{Types of interventions}

We will include studies of interventions that aim to improve medical students' interpersonal communication when undertaking medical consultations. Specifically, included interventions will target the communication tasks and skills associated with relationship building, gathering information, and planning and explaining, as well as specific tasks of communication such as listening, appropriate non-verbal communication, and providing closure (Makoul 2001b). Included interventions will be those focused on interpersonal communication in consultations with adult patients. This may be in dyadic (student - patient or student - carer) or triadic (student - patient - carer) scenarios. We will include the following comparisons:

- communication interventions to medical students versus no intervention;
- communication interventions to medical students versus usual training; and

- communication intervention A versus communication intervention B (both to medical students).

Where communication interventions are delivered as part of a larger complex intervention, we will only include studies that compare interpersonal communication as part of a complex intervention with the same complex intervention without interpersonal communication elements. This will enable us to determine the effects of the interpersonal communication element of the intervention.

\section{Types of outcome measures}

We will include outcomes assessed using:

1. observer ratings of interpersonal communication during student-patient consultations with real patients;

2. observer ratings of interpersonal communication during student-patient consultations with simulated patients;

3. observer ratings of interpersonal communication during video- or audio-taped student-patient consultations with simulated patients; or

4. scores obtained on surveys or questionnaires completed by real or simulated patients in relation to the learners' interpersonal communication.

It is likely that most of these assessments will be obtained through OSCEs. While such assessments are less than ideal in terms of the capacity to extrapolate from simulated situations to the actual behaviours used in real patient consultations, they are the most widely recognised and adopted proxy throughout medical education internationally. Given the inherent challenges of assessment in education interventions, these outcomes are regarded as best practice.

\section{Primary outcomes}

The primary outcome will be improved interpersonal communication at any point in a medical consultation (actual behaviour or patient satisfaction with said behaviour). Elements of interpersonal communication have been categorised as relating to relationship building, information gathering, planning and explaining, or patient appraisals, with an additional category of specific communication tasks to capture the elements which cannot be placed into a single one of these categories.

Trials that measure any of the following outcomes will be included, irrespective of whether they are regarded as primary or secondary outcomes in the trial itself. For all outcomes, we will assess terminology and measures used to ensure that any outcomes reported under different labels but pertaining to the measures below will be captured (e.g.: compassion taken to mean empathy, specific context-related questions taken to be part of information gathering). Primary outcomes for the review include: 
- relationship building; (e.g. rapport or relationship building, demonstrating empathic behaviour, communicating without judgement);

- information-gathering skills (e.g. appropriately using openended and closed questions, eliciting the patient's concerns or feelings);

- planning and explaining skills (e.g. giving appropriate information about a diagnosis and/or management plan, shared decision-making and acknowledging patient preferences);

- specific communication tasks (e.g. active listening, nonverbal communication, structuring the consultation, providing closure);

- simulated or real patient appraisals of the consultation (e.g. measures of satisfaction, therapeutic alliance, perceived support); and

- in line with Cochrane Methodological Expectations, adverse events (student complaints or referral to student welfare) will be included as a primary outcome.

We will not record secondary outcomes.

\section{Search methods for identification of studies}

\section{Electronic searches}

We will search the following electronic databases:

- Cochrane Central Register of Controlled Trials

(CENTRAL, The Cochrane Library, latest issue);

- MEDLINE OvidSP;

- Embase OvidSP;

- PsycINFO OvidSP;

- Educational Resource Information Centre, OvidSP.

We present the search strategy for MEDLINE (OvidSP) in Appendix 1. We will tailor strategies to other databases and report them in the review. Databases will be searched from their start date to the present.

\section{Searching other resources}

In addition to searching these databases, we will also:

- handsearch relevant journals in the field published since 2007 (e.g. Medical Education; BMC Medical Education, Medical Teacher);

- contact authors to clarify reported information and seek any unpublished data;

- review reference lists of relevant publications and systematic reviews;

- contact experts in the field for advice relating to other relevant studies;

- search trial registries:
- WHO International Clinical Trials Registry Platform

(ICTRP) (www.who.int/ictrp/en);

- ClinicalTrials.gov (clinicaltrials.gov);

- search relevant grey literature (e.g. dissertation/thesis portals); and

- search Google Scholar (e.g. review random sample of up to 200 citations).

\section{Data collection and analysis}

\section{Selection of studies}

We will combine search results in an Endnote database and remove duplicate records. Initial screening of titles and abstracts will be conducted independently by two people to determine which records meet the inclusion criteria. We will exclude studies that clearly do not meet the inclusion criteria. We will retrieve in full text any papers identified as potentially relevant by at least one person. Two people will independently screen full text articles for inclusion or exclusion. Two other people will act as independent arbiters to resolve disagreements regarding study inclusion. Studies that are excluded at this stage will be included in a 'Characteristics of excluded studies' table with reasons for exclusions. Three people will verify the final list of included studies. Disagreements regarding inclusion on the final list of studies will be resolved by these three authors. We will report the screening and selection process in an adapted PRISMA flow chart.

\section{Data extraction and management}

Two review authors will extract data independently from included studies. Any discrepancies will be resolved by discussion until consensus is reached, or through consultation with a third author. We will develop and pilot a data extraction form using the Cochrane Consumers and Communication Data Extraction Template (available at: cccrg.cochrane.org/author-resources). It will include the following items:

- general information: title, authors, source, publication status, date published, geographic location;

- methods: aims of study, study design, total study duration, inclusion/exclusion criteria, ethical approval, recruitment, informed consent, consumer involvement (in design of study/ intervention, delivery, evaluation, or interpretation of findings);

- participants: total number, characteristics (students - level in program), setting (on campus, clinical setting), age, sex, ethnicity, socioeconomic status, country, first language, participant evaluation of training, other available demographic information;

- intervention: theoretical framework, learning objectives, mode of delivery, timing within the medical program, number of intervention groups, number and frequency of sessions, 
congruence of language used in intervention with first-language of participants;

- facilitators: who delivered/facilitated the intervention, intervention-specific training undertaken by those delivering the intervention;

- outcomes: how the effectiveness of the intervention is assessed, category of outcome measures, outcome definition, validity/reliability of outcome measure, time points collected;

- results: number of participants allocated to each intervention group, sample size, missing participants, summary data for each intervention group, estimate of effects with $95 \%$ confidence interval and $P$ values, subgroup analyses, cost-utility of interventions; and

- funding source and other sources of bias.

Details of control conditions will be also extracted. Where insufficient detail is included in the publication, the authors of the publication will be contacted and asked to provide more information about the usual training condition.

All extracted data will be entered into RevMan 2014 by one review author, and will be checked for accuracy against the data extraction sheets by a second review author working independently.

\section{Assessment of risk of bias in included studies}

Two authors will independently assess the methodological risk of bias of included studies in accordance with theCochrane Handbook (Higgins 2011) and the Cochrane Consumers and Communication guidelines (Ryan 2013), which recommend the explicit reporting of the following individual elements for RCTs: random sequence generation; allocation sequence concealment; blinding (participants, personnel); blinding of outcome assessment (assessed for each outcome measure); completeness of outcome data, selective outcome reporting; and other sources of bias (we will consider the timing of outcome assessment and assessor relationship with student participants).

We will consider blinding separately for different outcomes where appropriate (for example, blinding may have the potential to differently affect subjective versus objective outcome measures). We will judge each item as being at high, low or unclear risk of bias as set out in the criteria provided by Higgins 2011, and provide a quote from the study report and a justification for our judgement for each item in the 'Risk of bias' table.

Studies will be deemed to be at the highest risk of bias if they are scored as at high or unclear risk of bias for either the sequence generation or allocation concealment domains, based on growing empirical evidence that these factors are particularly important potential sources of bias (Higgins 2011).

We will assess and report quasi-RCTs as being at a high risk of bias on the random sequence generation item of the 'Risk of bias' tool. For cluster RCTs we will also assess and report the risk of bias associated with an additional domain: selective recruitment of cluster participants.

\section{Measures of treatment effect}

The primary outcome measures identified by study authors that fall into each of the outcome categories will be the basis of measurement of treatment effect. In a post-hoc process, two authors will independently assign the outcomes reported in each included study to one of the outcome categories and a third author will be involved to resolve disagreements where required. Where multiple primary outcomes are identified within a category, we will rank the reported intervention effect estimates and select the median effect estimate. Where outcomes are reported for different time periods, we will use outcomes closest to the end on the intervention delivery.

We anticipate that outcomes will be measured with both dichotomous and continuous data.

\section{RCTs, quasi-RCTs and cluster RCTs}

We will analyse dichotomous data based on the number of events and the number of people assessed in the intervention and comparison groups. We will use these to calculate the risk ratio (RR) and $95 \%$ confidence interval (CI).

We will analyse continuous data based on the mean, standard deviation (SD) and number of people assessed for both the intervention and comparison groups to calculate mean difference (MD) and $95 \%$ CI. If the MD is reported without individual group data, we will use this to report the study results. If more than one study measures the same outcome using different tools, we will calculate the standardised mean difference (SMD) and 95\% CI using the inverse variance method in RevMan 2014.

\section{Unit of analysis issues}

If cluster RCTs are included we will check for unit of analysis errors. If errors are found, and sufficient information is available, we will reanalyse the data using the appropriate unit of analysis, by taking account of the intracluster correlation (ICC). We will obtain estimates of the ICC by contacting authors of included studies, or impute them using estimates from external sources. If it not possible to obtain sufficient information to reanalyse the data we will report effect estimates and annotate "unit of analysis error".

\section{Dealing with missing data}

We will attempt to contact study authors to obtain missing data (participant, outcome, or summary data). For participant data, we will, where possible, conduct analysis on an intention-to-treat basis; otherwise data will be analysed as reported. We will report on the levels of loss to follow-up and assess this as a source of potential bias.

For missing outcome or summary data we will impute missing data where possible and report any assumptions in the review. We will 
investigate, through sensitivity analyses, the effects of any imputed data on pooled effect estimates.

\section{Assessment of heterogeneity}

Where studies are considered similar enough (based on consideration of populations, interventions, primary outcome) to allow pooling of data using meta-analysis, we will assess the degree of heterogeneity by visual inspection of forest plots and by examining the $\mathrm{Chi}^{2}$ test for heterogeneity. Heterogeneity will be quantified using the $\mathrm{I}^{2}$ statistic. An $\mathrm{I}^{2}$ value of $50 \%$ or more will be considered to represent substantial levels of heterogeneity, but this value will be interpreted in light of the size and direction of effects and the strength of the evidence for heterogeneity, based on the $\mathrm{P}$ value from the $\mathrm{Chi}^{2}$ test (Higgins 2011).

Where we detect substantial clinical, methodological or statistical heterogeneity across included studies we will not report pooled results from meta-analysis but will instead use a narrative approach to data synthesis. In this event we will attempt to explore possible clinical or methodological reasons for this variation by grouping studies that are similar in terms of intervention features and methodological features to explore differences in intervention effects.

Note that when few trials are included in a meta-analysis, the $\mathrm{Chi}^{2}$ test has little power to detect heterogeneity. Therefore a nonsignificant result should not necessarily be interpreted as evidence of no heterogeneity and should instead be interpreted with care.

\section{Assessment of reporting biases}

We will assess reporting bias qualitatively based on the characteristics of the included studies (e.g. if only small studies that indicate positive findings are identified for inclusion), and if information that we obtain from contacting experts and authors or studies suggests that there are relevant unpublished studies.

If we identify sufficient studies (at least 10) for inclusion in the review we will construct a funnel plot to investigate small study effects, which may indicate the presence of publication bias. We will formally test for funnel plot asymmetry, with the choice of test made based on advice in Higgins 2011, and bearing in mind that there may be several reasons for funnel plot asymmetry when interpreting the results.

\section{Data synthesis}

Data synthesis will start with a systematic summary of all included studies and a narrative review of the findings. We will then determine if participants, interventions and outcome measures are comparable to allow statistical pooling. We will decide whether to meta-analyse data based on whether the interventions are similar enough in terms of participants, settings, intervention, comparison and outcome measures to ensure meaningful conclusions from a statistically pooled result. Due to the anticipated variability in the interventions and outcome measures of included studies, we will use a random-effects model for meta-analysis.

If we are unable to pool the data statistically using meta-analysis we will conduct a narrative synthesis of results. We will present the major outcomes and results, organised by the following categories:

- participant demographics;

- duration of intervention (intensity);

- timing of intervention within medical program/degree;

- methods of assessment/outcome measures; and

- intervention approach or mode of delivery.

Where studies compare more than one intervention, we will compare each separately to no intervention/control; and with one another.

\section{Subgroup analysis and investigation of heterogeneity}

Where there are appropriate available data, we will conduct subgroup analyses to determine variation in the effectiveness of communication interventions in relation to:

- intervention characteristics - setting, duration and timing of intervention;

- categories of outcome measures (relationship building, information gathering, explanation and planning, specific communication tasks); and

- intervention/training methods - face-to-face (small group with/without practice/demonstration with real or simulated patients, large group activities/lectures with/without demonstration by real or simulated patients), self-directed (online or written tasks).

If there are appropriate data, we will use random-effects meta-regression. This will enable us to estimate relative change in intervention effects for each subgroup variable. If the number of studies or volume of data do not warrant statistical subgroup analysis we will present subgroup analyses in narrative form.

\section{Sensitivity analysis}

We will undertake a sensitivity analysis based on the risk of bias. We will remove studies at high risk of bias and determine the impact on the pooled intervention effects.

\section{'Summary of findings' table}

We will prepare a 'Summary of findings' table to present the results of meta-analysis, based on the methods described in chapter 11 of the Cochrane Handbook for Systematic Reviews of Interventions (Schünemann 2011). We will present the results of meta-analysis for the major comparisons of the review, for each of the major primary outcomes, including potential harms, as outlined in Types of outcome measures. We will provide a source and rationale for each assumed risk cited in the table/s, and will use the GRADE 
system to rank the quality of the evidence using the GRADEprofiler (GRADEpro) software (Schünemann 2011). If meta-analysis is not possible, we will present results in a narrative 'Summary of findings' table format, such as that used by Chan 2011 .

\section{Ensuring relevance to decisions in health care}

The author team consists of a number of international experts who will contribute specific sections of the review and provide input regarding the role of interpersonal communication in the advancement of patient-centred health care. We are establishing an advisory group of medical students representing international medical student associations (including Australia, Europe, the UK, and North America), as well as simulated patient groups from the UK and Australia. To date, members have reviewed the protocol to ensure that the objectives, inclusion/exclusion criteria, outcome measures, and proposed data extraction items are appropriate and adequately reflect their experiences and priorities. Existing members of the group have also reviewed the language and definitions of terms used to ensure adequate representation. The full group will conduct similar review processes at several stages during preparation of the review. The group's objective is to provide feedback on how the review reflects the real-world importance of providing interpersonal communication training to medical students.

\section{ACKNOW LEDGEMENTS}

We thank the editors and staff of Cochrane Consumers and Communication, particularly our contact editor Dr Bronwyn Hemsley, for their input to this protocol.

\section{RE F ER E N C E S}

\section{Additional references}

\section{Alliger 1989}

Alliger GM, Janak EA. Kirkpatrick's levels of training criteria: thirty years later. Personnel Psychology 1989;42(2): $331-42$.

\section{Aper 2015}

Aper L, Veldhuijzen W, Dornan T, van de Ridder M, Koole $\mathrm{S}$, Derese A, et al. "Should I prioritize medical problem solving or attentive listening?": the dilemmas and challenges that medical students experience when learning to conduct consultations. Patient Education and Counseling 2015;98 (1):77-84.

\section{Aspegren 1999}

Aspegren K. BEME Guide No. 2: Teaching and learning communication skills in medicine-a review with quality grading of articles. Medical Teacher 1999;21(6):563-70.

Australian Medical Council 2012

Australian Medical Council Limited. Standards for assessment and accreditation of primary medical programs by the Australian Medical Council 2012. www.amc.org.au/ joomla-files/images/Accreditation/FINAL-Standards-andGraduate-Outcome-Statements-20-December-2012.pdf. Kingston, ACT, 2012 (accessed prior to 6 October 2016).

\section{Benbassat 2009}

Benbassat J, Baumal R. A proposal for overcoming problems in teaching interviewing skills to medical students. Advances in Health Sciences Education 2009;14(3):441-50.

\section{Berkhof 2011}

Berkhof M, van Rijssen HJ, Schellart AJM, Anema JR, van der Beek AJ. Effective training strategies for teaching communication skills to physicians: an overview of systematic reviews. Patient Education and Counseling 2011; 84(2):152-62.
Boon 1998

Boon H, Stewart M. Patient-physician communication assessment instruments: 1986 to 1996 in review. Patient Education and Counseling 1998;35(3):161-76.

Burleson 2010

Burleson BR. The nature of interpersonal communication: a message-centred approach. In: Berger CR, Roloff ME, Roskos-Ewoldsen DR editor(s). The Handbook of Communication Science. 2nd Edition. California: SAGE, 2010.

CCCG 2013

Cochrane Consumers and Communication Group. Standard protocol text and additional guidance for review authors. cccrg.cochrane.org 2013 (accessed prior to 6 October 2016).

\section{Chan 2011}

Chan RJ, Webster J, Marquart L. Information interventions for orienting patients and their carers to cancer care facilities. Cochrane Database of Systematic Reviews 2011, Issue 12. [DOI: 10.1002/14651858.CD008273.pub2]

Cole 2013

Cole SA, Bird J. The Medical Interview: The Three Function Approach. 3rd Edition. Philadelphia (PA): Elsevier Health Sciences, 2013.

\section{Cook 2010}

Cook DA, Erwin PJ, Triola MM. Computerized virtual patients in health professions education: a systematic review and meta-analysis. Academic Medicine 2010;85(10): 1589-602.

Deveugele 2005 Deveugele M, Derese A, De Maesschalck S, Willems S, Van Driel M, De Maeseneer J. Teaching communication skills to medical students, a challenge in the curriculum?. Patient Education and Counseling 2005;58(3):265-70. 


\section{Duffy 2004}

Duffy FD, Gordon GH, Whelan G, Cole-Kelly K, Frankel R, Buffone N, et al. Participants in the American Academy on Physician, Patient's Conference on Education, Evaluation of Competence in Communication, Interpersonal Skills. Assessing competence in communication and interpersonal skills: the Kalamazoo II report. Academic Medicine 2004;79 (6):495-507.

Duke 2013

Duke P, Frankel RM, Reis S. How to integrate the electronic health record and patient-centered communication into the medical visit: a skills-based approach. Teaching and Learning in Medicine 2013;25(4):358-65.

General Medical Council 2015 General Medical Council. Outcomes for graduates. www.gmc-uk.org/education/undergraduate/undergrad_ outcomes.asp 2015 (accessed prior to 6 October 2016).

Gregory 2007

Gregory J. Conceptualising consumer engagement: a review of the literature. Australian Institute of Health Policy Studies 2007.

\section{Hargie 2010}

Hargie O, Boohan M, McCoy M, Murphy P. Current trends in communication skills training in UK schools of medicine. Medical Teacher 2010;32(5):385-91.

\section{Hargie 2011}

Hargie O. Skilled Interpersonal Communication: Research, Theory and Practice. 5th Edition. New York (NY): Routledge, 2011.

\section{Hartley 1999}

Hartley P. Interpersonal Communication. 2nd Edition. New York (NY): Routledge (Taylor and Francis), 1999.

Health Professions Council of South Africa 2014

Health Professions Council of South Africa. Core competencies for undergraduate students in clinical associate, dentistry and medical teaching and learning programmes in South Africa. www.hpcsa.co.za/ PBMedicalDental/Education 2014 (accessed prior to 6 October 2016).

\section{Higgins 2011}

Higgins JPT, Green S (editors). Cochrane Handbook for Systematic Reviews of Interventions Version 5.1.0 [updated March 2011]. The Cochrane Collaboration, 2011. Available from www.cochrane-handbook.org.

\section{Hoffman 2004}

Hoffman M, Ferri J, Sison C, Roter D, Schapira L, Baile W. Teaching communication skills: an AACE survey of oncology training programs. Journal of Cancer Education 2004;19(4):220-4.

Kaufman 2003

Kaufman DM. Applying educational theory in practice. BMJ 2003;326(7382):213-6.
Keller 1994

Keller V, Carroll JG. A new model for physician-patient communication. Patient Education and Counseling 1994; 23:131-40.

Kirkpatrick 1996

Kirkpatrick D. Revisiting Kirkpatrick's four-level model. Training and Development 1996;50(1):54-9.

\section{Kurtz 1998}

Kurtz S, Silverman J, Draper J. Teaching and Learning Communication Skills in Medicine. Abingdon, UK: Radcliffe Medical Press, 1998.

\section{Kurtz 2003}

Kurtz S, Silverman J, Benson J, Draper J. Marrying content and process in clinical method teaching: enhancing the Calgary-Cambridge guides. Academic Medicine 2003;78(8): 802-9.

\section{Laidlaw 2009}

Laidlaw A, Guild S, Struthers J. Graduate attributes in the disciplines of Medicine, Dentistry and Veterinary Medicine: a survey of expert opinions. BMC Medical Education 2009; 9:28.

\section{Lanken 2015}

Lanken PN, Novack DH, Daetwyler C, Gallop R, Landis JR, Lapin J, et al. Efficacy of an internet-based learning module and small-group debriefing on trainees' attitudes and communication skills toward patients with substance use disorders: results of a cluster randomized controlled trial. Academic Medicine 2015;90(3):345-54. [DOI: 10.1097/ACM.0000000000000506]

\section{Lipkin 1995}

Lipkin Jr M, Kaplan C, Clark W, Novack DH. Teaching medical interviewing: the Lipkin model. In: Lipkin M Jr, Putnam SM, Lazare A editor(s). The Medical Interview: Clinical Care, Education, and Research. 1st Edition. New York, NY: Springer, 1995:422-35.

\section{Lumma-Sellenthin 2009}

Lumma-Sellenthin A. Talking with patients and peers: medical students' difficulties with learning communication skills. Medical Teacher 2009;31(6):528-34.

\section{Macdonald 2002}

Macdonald G. Transformative unlearning: safety, discernment and communities of learning. Nursing Inquiry 2002;9(3):170-8.

\section{MacDonald-Wicks 2012}

MacDonald-Wicks L, Levett-Jones T. Effective teaching of communication to health professional undergraduate and postgraduate students: a systematic review. JBI Library of Systematic Reviews 2012;10(28 Suppl):S172-83.

\section{Maguire 1986}

Maguire P, Fairbairn S, Fletcher C. Consultation skills of young doctors: I - benefits of feedback training in interviewing as students persist. British Medical Journal 1986;292(6535):1573-6.

Maguire 2002

Maguire P, Pitceathly C. Key communication skills and how to acquire them. BMJ 2002;325(7366):697-700. 


\section{Makoul 1998}

Makoul G. Communication research in medical education. In: Jackson L, Duffy BK editor(s). Health Communication Research: A Guide to Developments and Directions. Wesport, CT: Greenwood Press, 1998:17-35.

\section{Makoul 2001a}

Makoul G. The SEGUE framework for teaching and assessing communication skills. Patient Education and Counseling 2001;45(1):23-34.

\section{Makoul 2001b}

Makoul G. Essential elements of communication in medical encounters: the Kalamazoo consensus statement. Academic Medicine 2001;76(4):390-3.

\section{Mead 2002}

Mead N, Bower P. Patient-centred consultations and outcomes in primary care: a review of the literature. Patient Education and Counseling 2002;48(1):51-61.

\section{Naugle 2000}

Naugle KA, Naugle LB, Naugle RJ. Kirkpatrick's evaluation model as a means of evaluating teacher performance. Education 2000;121(1):135.

\section{NHS 2010}

National Health Service (UK). Liberating the NHS: No decision about me, without me - Further consultation on proposals to secure shared decision-making. www.gov.uk/ government/uploads/system/uploads/attachment_data/ file/216980/Liberating-the-NHS-No-decision-about-mewithout-me-Government-response.pdf 2010 (accessed prior to 6 October 2016).

\section{Novack 1992}

Novack DH, Dube C, Goldstein MG. Teaching medical interviewing: a basic course on interviewing and the physician-patient relationship. Archives of Internal Medicine 1992;152(9):1814-20.

Peterson 1992

Peterson MC, Holbrook JH, Von Hales D, Smith NL, Staker LV. Contributions of the history, physical examination, and laboratory investigation in making medical diagnoses. Western Journal of Medicine 1992;156(2):163-5.

Pinto 2012

Pinto RZ, Ferreira ML, Oliveira VC, Franco MR, Adams $\mathrm{R}$, Maher CG, et al. Patient-centred communication is associated with positive therapeutic alliance: a systematic review. Journal of Physiotherapy 2012;58(2):77-87.

Pollak 2011

Pollak KI, Alexander SC, Tulsky JA, Lyna P, Coffman CJ, Dolor RJ, et al. Physician empathy and listening: associations with patient satisfaction and autonomy. Journal of the American Board of Family Medicine 2011;24(6): $665-72$.

Rees 2002

Rees CE, Sheard CE, McPherson AC. A qualitative study to explore undergraduate medical students' attitudes towards communication skills learning. Medical Teacher 2002;24 (3):289-93.
RevMan 2014 [Computer program]

Nordic Cochrane Centre, The Cochrane Collaboration. Review Manager (RevMan). Version 5.3. Copenhagen: Nordic Cochrane Centre, The Cochrane Collaboration, 2014.

Richardson 2001

Richardson WC et al. (Committee on Quality of Health Care in America, Institute of Medicine). Crossing the Quality Chasm: A New Health System for the 21st Century. In: Briere R editor(s). Institute of Medicine. 1st Edition. Vol. 1, Washington DC: National Academies Press, 2001.

\section{Royston 1997}

Royston V. How do medical students learn to communicate with patients? A study of fourth-year medical students' attitudes to doctor-patient communication. Medical Teacher 1997;19(4):257-62.

Ryan 2013

Ryan R, Hill S, Prictor M, McKenzie J, Cochrane Consumers, Communication. Study quality guide. cccrg.cochrane.org/authorresources May 2013 (accessed prior to 6 October 2016).

\section{Schünemann 2011}

Schünemann HJ, Oxman AD, Higgins JPT, Vist GE, Glasziou P, Guyatt GH. Chapter 11: Presenting results and 'Summary of findings' tables. In: Higgins JPT, Green $S$ (editors), Cochrane Handbook for Systematic Reviews of Interventions Version 5.1.0 [updated March 2011]. The Cochrane Collaboration, 2011. Available from www.cochrane-handbook.org.

Silverman 2013

Silverman J, Kurtz SM, Draper J, van Dalen J, Platt FW. Skills for Communicating with Patients. 3rd Edition. Oxford (UK): Radcliffe, 2013.

\section{Simpson 1991}

Simpson M, Buckman R, Stewart M, Maguire P, Lipkin $\mathrm{M}$, Novack D, et al. Doctor-patient communication: the Toronto consensus statement. BMJ 1991;303(6814): 1385-7.

\section{Smidt 2009}

Smidt A, Balandin S, Sigafoos J, Reed VA. The Kirkpatrick model: a useful tool for evaluating training outcomes. Journal of Intellectual and Developmental Disability 2009;34 (3):266-74.

\section{Smith 2007}

Smith S, Hanson JL, Tewksbury LR, Christy C, Talib NJ, Harris MA, et al. Teaching patient communication skills to medical students: a review of randomized controlled trials. Evaluation \& the Health Professions 2007;30(1):3-21.

\section{Stewart 1995a}

Stewart M, Belle Brown J, Weston WW, McWhinney IR, McWilliam CL, Freeman TR. Patient-centred Medicine: Transforming the Clinical Method. Thousand Oaks, CA: SAGE, 1995. 


\section{Stewart 1995b}

Stewart MA. Effective physician-patient communication and health outcomes: a review. Canadian Medical

Association Journal 1995;152(9):1423-33.

\section{Stewart 2001}

Stewart M. Towards a global definition of patient centred care: the patient should be the judge of patient centred care. BMJ 2001;322:444.

\section{Street 2009}

Street RL, Makoul G, Arora NK, Epstein RM. How does communication heal? Pathways linking clinician-patient communication to health outcomes. Patient Education and Counseling 2009;74(3):295-301.

\section{Thistlethwaite 1999}

Thistlethwaite JE, Jordan JJ. Patient-centred consultations: a comparison of student experience and understanding in two clinical environments. Medical Education 1999;33(9): 678-85.

\section{Thompson 2016}

Thompson L, Howes C, McCabe R. Effect of questions used by psychiatrists on therapeutic alliance and adherence. British Journal of Psychiatry 2016;209(1):40-7.

\section{Van Nuland 2005}

Van Nuland M, Hannes K, Cools F, Goedhuys J.

Educational interventions for improving the communication skills of general practice trainees in the clinical consultation. Cochrane Database of Systematic Reviews 2005, Issue 4. [DOI: 10.1002/14651858.CD005559]

\section{Windover 2014}

Windover AK, Boissy A, Rice TW, Gilligan T, Velez VJ, Merlino J. The REDE model of healthcare communication: optimizing relationship as a therapeutic agent. Journal of Patient Experience 2014;1 (1):8-13.

\section{Zolnierek 2009}

Zolnierek KB, DiMatteo MR. Physician communication and patient adherence to treatment: a meta-analysis. Medical Care 2009;47(8):826-34.

* Indicates the major publication for the study

\section{A P P E N D I CES}

\section{Appendix I. MEDLINE search strategy}

1. students medical/

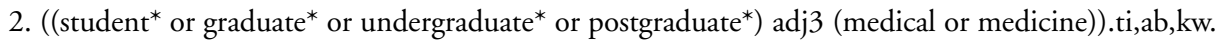

3. education medical/

4. education medical undergraduate/

5. clinical clerkship/

6. education medical graduate/

7. (medic* adj3 (education or school* ${ }^{*}$ or course* or curricul*)).ti,ab,kw.

8. or/1-7

9. communication/

10. (communicat* adj3 (skill* or competen*)).tw.

11. ((patient or client or family) adj (cent?red or focus?ed or tailored)).tw.

12. patient centered care/

13. interpersonal relations/

14. interpersonal.tw.

15. cultural competency/

16. (cultur* adj3 (competenc* or understanding or knowledg* or sensitiv* or aware* or respons* or appropriate* or acceptab* or safe* or humility)).ti,ab,kw.

17. (intercultural ${ }^{*}$ or inter-cultural* ${ }^{*}$ or transcultural* ${ }^{*}$ trans-cultural ${ }^{*}$ or cross-cultural* ${ }^{*}$ or crosscultural*).ti,ab,kw.

18. empathy/

19. (therapeutic alliance or empath* or bad news or listening skill*).tw.

20. physician patient relations/

21. ((physician or doctor or gp or general practitioner) adj1 (patient or client)).tw.

22. ((patient* or client*) adj3 (interact* or relations* or deal* with or rapport)).tw.

23. (relations* adj3 build*).ti,ab,kw.

Interventions for improving medical students' interpersonal communication in medical consultations (Protocol)

Copyright @ 2016 The Cochrane Collaboration. Published by John Wiley \& Sons, Ltd. 
24. exp "referral and consultation"/

25. office visits/

26. interviews as topic/

27. negotiating/

28. ((ask* adj3 question*) or questioning or explain* or discuss or discussing or closure).ti,ab,kw.

29. (information adj3 gather*).ti,ab,kw.

30. trust/

31. (verbal or nonverbal or non-verbal or smiling or negotiat* or trust or hope or friendl* or warmly or cultural* ${ }^{*}$ spiritual* $^{*}$ or comforting or supportive*).tw.

32. (consult* or interview* skill*).tw.

33. ((shar* or join* or concordan* or participat*) adj3 decision making).tw.

34. or/26-33

35. (patient* or client* or skill* or competen*).tw.

36. 34 and 35

37. ((medical or clinical) adj encounter*).tw.

38. exp medical history taking/

39. (history taking or anamnesis).tw.

40. or/9-25,36-39

41.8 and 40

42. randomized controlled trial.pt.

43. controlled clinical trial.pt.

44. randomized.ab.

45. placebo.ab.

46. drug therapy.fs.

47. randomly.ab.

48. trial.ab.

49. groups.ab.

50. or/42-49

51.41 and 50

\section{CONTRIBUTIONSOFAUTHORS}

Erica James and Pamela Snow conceived of the review. All authors have contributed to the design of the review and writing the protocol. Conor Gilligan has led the writing of the protocol and will coordinate the review process (guarantor). Conor Gilligan, Erica James, Sue Outram, Bernadette Ward, Martine Powell, Chris Lonsdale, Pam Harvey, Timothy Regan, and Marita Lynagh will all be involved with elements of data collection, analysis, interpretation of findings and writing the review. Anne Cushing and Jonathan Silverman will provide general advice on the review and assist with interpretation of results.

\section{DECLARATIONS OF INTEREST}

- Conor Gilligan: None known.

- Erica L James: None known.

- Pamela Snow: None known.

- Sue Outram: None known.

- Bernadette M Ward: None known

- Martine Powell: None known.

- Chris Lonsdale has received consultancy fees from commercial agencies on health promotion products. His research is supported by grants from government and philanthropic agencies.

Interventions for improving medical students' interpersonal communication in medical consultations (Protocol)

Copyright (๑) 2016 The Cochrane Collaboration. Published by John Wiley \& Sons, Ltd. 
- Anne M Cushing: None known.

- Jonathan Silverman has worked at the forefront of the development of communication skills teaching in undergraduate medical education for the last 20 years and has recently retired from the School of Clinical Medicine, University of Cambridge, where he led the undergraduate communication programme. He receives royalties from two books that he wrote about healthcare communication and he travels as president of the European Association for Communication in Healthcare.

- Tim Regan: None known.

- Pam Harvey: None known.

- Marita C Lynagh: None known.

\section{NOTES}

This protocol is based on standard text and guidance provided by Cochrane Consumers and Communication (CCCG 2013). 